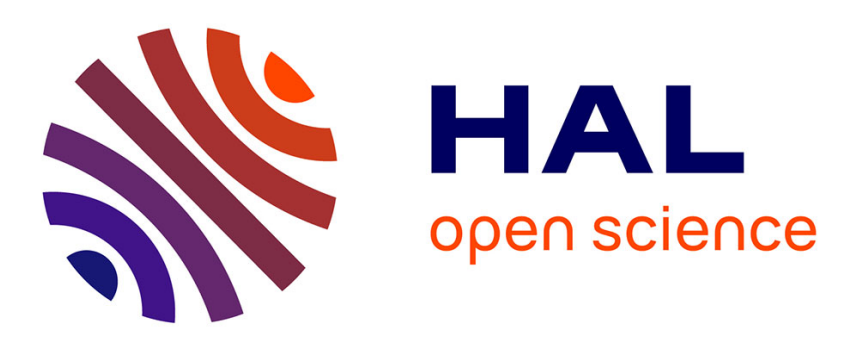

\title{
Les métamorphoses du mot: la citation de vers chinois comme sujet de composition de poèmes japonais, Waka
}

Michel Vieillard-Baron

\section{To cite this version:}

Michel Vieillard-Baron. Les métamorphoses du mot: la citation de vers chinois comme sujet de composition de poèmes japonais, Waka. Extrême-Orient Extrême-Occident, 1995, 17 (17), pp.67 - 90. 10.3406/oroc.1995.1005 . hal-01686751

\section{HAL Id: hal-01686751 \\ https://hal-inalco.archives-ouvertes.fr/hal-01686751}

Submitted on 17 Jan 2018

HAL is a multi-disciplinary open access archive for the deposit and dissemination of scientific research documents, whether they are published or not. The documents may come from teaching and research institutions in France or abroad, or from public or private research centers.
L'archive ouverte pluridisciplinaire HAL, est destinée au dépôt et à la diffusion de documents scientifiques de niveau recherche, publiés ou non, émanant des établissements d'enseignement et de recherche français ou étrangers, des laboratoires publics ou privés. 


\title{
Les métamorphoses du mot : la citation de vers chinois comme sujet de composition de poèmes japonais, Waka
} Michel Vieillard-Baron

\begin{abstract}
The metaporphosis of the word : the quotation of Chinese verses as themes for the composition of Japanese Waka poems

At the end of the ninth century the Japanese poet Ôe no Chisato wrote Japanese poems waka which took Chinese verses as themes (mostly taken from those of the famous Tang poet Bai Juyi). He thus initiated a new style of composition. In 1218 a Japanese poet Jien chose one hundred verses/themes from Bai Juyi's works and asked Fujiwara no Teika to compose on these themes, as he was doing himself. Among the themes chosen by Ôe, Jien and Teika, seven were common. Their analysis allows us to retrace the evolution of this innovative style of composition and to define what motivated it.
\end{abstract}

\section{Résumé}

À la fin du IXe siècle, le poète japonais Ôe no Chisato composa des " poèmes japonais » waka, en prenant pour sujets des vers extraits d'oeuvres chinoises, en particulier celles du célèbre poète des Tang, .Bai Juyi. II inaugurait ainsi un nouveau mode de composition.

Plus de deux siècles plus tard, le poète japonais Jien choisit cent vers sujets extraits de poèmes de Bai Juyi et demanda à Fujiwara no Teika de composer, tout comme lui, sur ces sujets. On trouve sept sujets communs parmi ceux qu'ont choisis Ôe no Chisato et Jien. L'analyse des poèmes composés par ces deux poètes et Teika sur les sept sujets nous permet de retracer l'évolution de ce mode de composition original et d'en définir la motivation.

\section{Citer ce document / Cite this document :}

Vieillard-Baron Michel. Les métamorphoses du mot : la citation de vers chinois comme sujet de composition de poèmes japonais, Waka. In: Extrême-Orient, Extrême-Occident, 1995, n¹7. Le travail de la citation en Chine et au Japon. pp. 6790.

doi : 10.3406/oroc.1995.1005

http://www.persee.fr/doc/oroc_0754-5010_1995_num_17_17_1005

Document généré le 16/10/2015 


\section{Les métamorphoses du mot : la citation de vers chinois comme sujet de composition de poèmes japonais, Waka}

Michel Vieillard-Baron*

À la fin du IX $x^{\mathrm{e}}$ siècle (en 894 ou 896), le poète Ôe no Chisato ${ }^{1}$ compila sur l'ordre de l'empereur Uda un recueil de ses poèmes personnels. Ce recueil, qui contient cent vingt-six poèmes japonais, $w a k a^{2}$, a pour principale caractéristique de présenter cent seize œuvres composées en prenant pour sujet des vers extraits de poèmes chinois de l'époque des Tang (618-907). Ôe no Chisato s'inspira sans doute pour ce mode de composition d'une pratique connue dès le $\mathrm{VI}^{e}$ siècle en Chine; la composition de poèmes chinois, prenant pour sujet un ou plusieurs vers extraits d'autres poèmes chinois. Nous savons que les Japonais empruntèrent à la Chine son système d'écriture, et que, même après l'invention et la diffusion au cours des VIII et $\mathrm{IX}^{e}$ siècles de syllabaires permettant de transcrire de façon plus adéquate la langue japonaise, l'utilisation du chinois comme langue écrite ne fut jamais abandonnée. Les Japonais ne limitèrent pas leur emprunt au seul système d'écriture chinois. Ils importèrent également les pratiques lettrées, et parmi celles-ci, la composition de poèmes en chinois, kanshi. Si l'époque précise à laquelle apparurent au Japon des compositions poétiques en langue chinoise ne nous est pas connue, il semble bien que le plus ancien exemple attesté de "poème chinois » composé au Japon sur un vers/sujet extrait d'un poème chinois soit une œuvre de l'empereur Saga (règne 809-823) ${ }^{3}$. Ce mode de composition, appelé en japonais kudaishi, 匀题 pour sujet un vers chinois », se développa au Japon à la fin du IX ${ }^{e}$ siècle et au début du $\mathrm{X}^{\mathrm{e}}$ siècle, sous l'impulsion de poètes de premier plan tels que Shimada no Tadaomi (mort en 892 ?) - qui composa uniquement en chinois - et Sugawara no Michizane (mort en 903) qui excellait dans les compositions en chinois et en japonais. 
C'est de ce mode de composition que s'inspira Ôe no Chisato pour écrire les poèmes de son recueil. Ce recueil, intitulé Chisato-shû, Recueil de poèmes de Chisato, ou Kudai-waka, Poèmes japonais ayant pour sujet des vers chinois, est constitué, rappelons-le, de cent vingtsix poèmes : vingt-et-un poèmes de printemps, quatorze poèmes d'été, vingt-et-un poèmes d'automne, douze poèmes d'hiver, onze poèmes sur le vent et la lune, treize poèmes sur des excursions, onze poèmes de séparation, vingt-et-un poèmes de plaintes (les dix derniers ne sont toutefois pas composés sur des vers/sujets chinois) ${ }^{4}$. Chisato emprunta, pour les utiliser comme sujets, des vers de Yuan Zhen (779831), Zhang Xiaobiao (première moitié du $\mathrm{IX}^{\mathrm{e}} \mathrm{s}$.), Shangguan Yi (616 ?-664), mais surtout de Bai Juyi (772-846) qui à lui seul compte soixante-quatorze vers/sujets cités. Bai Juyi, ou Bo Juyi (en japonais Haku Kyoi ou, plus couramment Haku Rakuten), fut connu très tôt au Japon (peut-être même de son vivant), et son influence sur la littérature japonaise est considérable ${ }^{5}$. Il n'est dès lors pas étonnant que, plus de deux siècles après Ôe no Chisato, le poète japonais Jien ait encore choisi des vers de Bai Juyi comme sujet de composition de waka. En effet, la sixième année de Kenpô, c'est-à-dire en 1218, Jien (11551225 ), religieux et homme de lettres ${ }^{6}$, choisit parmi les poèmes de Bai Juyi des vers susceptibles de servir de sujets de composition pour une séquence de cent poèmes ${ }^{7}$. Il classa ensuite ces vers en fonction des thèmes qu'il souhaitait traiter, à savoir, quinze sujets pour les poèmes de printemps, dix pour les poèmes d'été, quinze pour les poèmes d'automne, dix pour les poèmes d'hiver, cinq pour les poèmes d'amour, cinq pour les poèmes sur la vie érémitique en montagne, sanka, cinq pour les poèmes sur des lieux habités autrefois, furusato, dix pour les poèmes sur la vie paisible, kankyo, dix pour les poèmes de plaintes, jukkai, dix pour les poèmes sur l'impermanence, mujô, et enfin, cinq pour les poèmes sur les enseignements de Bouddha, hômon. Il convia ensuite l'un des plus fameux poètes de son temps, Fujiwara no Teika (1162-1241) ${ }^{8}$, à composer sur ces mêmes vers/sujets 9 .

Entre le recueil de Ôe no Chisato et les séquences de Jien et Teika, plus de deux siècles de distance... et sept sujets communs! C'est sur ces sept vers/sujets et sur les poèmes qu'ils suscitèrent que portera la présente étude. Par l'analyse des poèmes que ces trois hommes composèrent, nous tenterons de saisir l'évolution, au cours de ces deux 
siècles, d'un mode de composition original, et de définir l'attitude de ces trois poètes face à un même matériau: un vers/sujet chinois.

Les premières œuvres que nous allons examiner furent composées sur un vers/sujet extrait du poème de Bai Juyi intitulé «Fleuve au printemps », chun jiang, (livre 18, n. 1159) ${ }^{10}$ :

I - «Convié par le chant du rossignol, je viens sous les fleurs», ying sheng you yin lai hua xia.

Sur ce vers/sujet Chisato composa (n. 2) ${ }^{11}$ :

$\begin{array}{ll}\text { Uguhisu no } & \text { Convié } \\ \text { nakitsuru kowe ni } & \text { par le chant } \\ \text { sasoharete } & \text { du rossignol, } \\ \text { hana no shita ni zo } & \text { c'est sous les fleurs (de prunier) } \\ \text { ware ha kinikeri. } & \text { que moi, je suis venu. }\end{array}$

Nous remarquons tout de suite que Chisato a conservé dans son poème tous les éléments du vers/sujet: le chant, le rossignol, convier, venir, sous, et enfin les fleurs (de prunier). Nous ne relevons d'autre différence entre le vers chinois et le poème japonais, que la présence dans ce dernier du pronom personnel «moi », et par là-même, sémantiquement, un renforcement de la présence du personnage dans son œuvre.

Examinons maintenant le poème de Jien composé sur le même sujet (n. 1912):

$\begin{array}{ll}\text { Uchikaheshi } & \text { De nouveau } \\ \text { uguhisu sasohu } & \text { soyons celui } \\ \text { mi to naramu } & \text { qui convie le rossignol. } \\ \text { koyohi wa hana no } & \text { Cette nuit, sous les fleurs de prunier, } \\ \text { shita ni yadorite } & \text { sans doute, je logerai. }\end{array}$

Jien a conservé du sujet le «rossignol», « convier», «sous», «les fleurs de prunier», mais rejeté le «chant» et le verbe «venir». Il a par ailleurs introduit l'impression de répétition signifiée par l'expression «de nouveau», et l'idée de «loger» (sous les fleurs), qui ne figuraient 
pas dans le vers chinois. Jien a également opéré un travail sur le sens du vers chinois. La tradition dont se fait l'écho ce vers/sujet veut que le rossignol chante dans les pruniers en fleur au début du printemps. Jien place son poème au moment de la saison où les pruniers sont déjà en fleur, toutefois le rossignol n'a pas encore commencé à chanter. C'est le poète, et là réside, nous semble-t-il, toute l'originalité de ce poème, qui chaque année, attiré par la beauté des fleurs de prunier, vient les contempler sitôt écloses, dormir sous leurs rameaux, et invite le rossignol à chanter.

Examinons maintenant le poème de Teika (n. 412):

Koromo de ni

midarete otsuru

hana no e ya

sasoharete kitsuru

uguhisu no kowe

\author{
Sur ma manche \\ tombent en désordre \\ des rameaux fleuris, \\ (par eux) convié, je suis venu, \\ (et j'entends) le chant du rossignol.
}

Teika a également conservé du sujet les fleurs de prunier (exprimées dans son poème par «rameaux fleuris»), les verbes venir et convier, le rossignol et son chant. Il a exprimé de façon détournée la position sous les fleurs, en disant que les rameaux tombent. Enfin, il a rajouté la manche qui ne figurait pas dans le vers chinois. Teika, tout comme Jien, a transformé le sens du vers/sujet. Ce n'est pas le rossignol qui convie le poète, ce sont les pruniers en fleur. C'est alors qu'il les contemple que se fait entendre le chant du rossignol, comme par pure coïncidence.

Les exemples que nous allons à présent examiner, également des poèmes de printemps, furent composés sur un vers/sujet extrait du poème de Bai Juyi intitulé «En réponse (au poème) que grand frère Shuda m'envoya», Chou ge Shuda xian zeng; (livre 13, n.616):

II - «(Assis) sous les fleurs de prunier nous oublions de rentrer, à cause de la beauté du paysage», Hua xia wang gui yin mei jing.

Sur ces vers Chisato composa (n. 14):

Hana wo mite

kaheramu koto wo
Contemplant les fleurs, on oublie de rentrer 
wasururu wa

irokoki kaze ni

yorite narikeri chez soi,

c'est à cause du vent

à la couleur profonde.

Chisato a gardé du sujet les fleurs (de prunier), rentrer chez soi, oublier, et l'expression «à cause». Il n'a pas exprimé directement le «sous les fleurs» du vers chinois, qu'il jugeait sans doute implicite dans l'expression «contempler les fleurs». Il a par ailleurs modifié la cause de l'oubli de rentrer chez soi. Il s'agissait de la beauté du paysage dans le vers chinois. Dans son poème, la cause est «le vent à la couleur profonde ». Nos recherches ne nous ont pas permis de trouver un précédent chinois ou japonais à cette étrange expression. «La couleur du vent» signifie dans la tradition chinoise: le temps qu'il fait, l'air, l'allure de quelqu'un, la couleur du ciel ou encore la situation. L'adjectif «profond» nong (jap. koi) signifie: épais, sombre, chargé de rosée, beau. L'expression "vent à la couleur profonde", vraisemblablement créée par Chisato, signifie «un ciel chargé », «un ciel ayant de belles couleurs ». Par l'emploi de cette expression, Chisato a voulu susciter un effet de surprise chez le lecteur.

Sur ce même sujet Jien composa (n. 1915):

Haru no yama ni

kasumi no sode wo

katashikite

ikuka ni narinu

hana no shita bushi
Dans la montagne au printemps, ma manche de brume

j'étale, couché sous un (prunier) en fleur, je ne sais combien de temps j'ai passé.

Dans son poème, Jien a gardé du sujet l'oubli de rentrer chez soi (exprimé indirectement par l'expression «je ne sais combien de temps j'ai passé »), et la localisation sous les fleurs de prunier. Il n'a, par ailleurs, exprimé ni la cause, ni la beauté du paysage (jugée sans doute redondante avec l'évocation des pruniers). Ce poème est l'occasion pour Jien d'utiliser une image insolite: la manche de brume qu'il étale. Étaler la manche de son vêtement signifie dans la littérature japonaise classique «dormir seul », sans l'être aimé, en d'autres termes: un sommeil solitaire et triste. L'expression «manche de brume», apparue vraisemblablement à l'époque de Jien, et vite devenue à la mode, est due à la similitude entre la manche d'un vêtement et une bande de 
brume. Jien combine ces deux expressions dans son poème pour lui conférer une couleur originale.

Voyons maintenant le poème de Teika (n. 415):

$\begin{array}{ll}\text { Toki shimo are } & \text { C'est sans doute le fait de la saison, } \\ \text { koshiji wo isogu } & \text { mais, des oies sauvages } \\ \text { karigane no } & \text { qui vers le nord s'empressent, } \\ \text { kokoro shirarenu } & \text { je ne puis comprendre le sentiment, } \\ \text { hana no moto kana } & \text { au pied des pruniers en fleur! }\end{array}$

Teika n'a gardé du sujet que la localisation sous les pruniers en fleur. Celle-ci lui permet de bâtir un poème dans lequel il exprime son incompréhension à l'égard des oies sauvages qui rentrent vers le nord au début du printemps, indifférentes à la beauté des pruniers en fleur (que lui est en train d'admirer).

Les exemples que nous allons examiner à présent sont également des poèmes de printemps. Le vers/sujet est extrait du poème de Bai Juyi (livre 16, n. 921) intitulé : «Fin de printemps, poème offert au maître de zen $(\mathrm{Zi})$ Chang, lorsque je montais à la tour sud du temple Dayun.» Wan chun deng Dayunsi nan lou zeng chang chan shi:

III - «Dans une année, les jours de printemps sont peu nombreux/ Dans le monde, les gens qui souffrent sont légion», Sui shi chun ri shao /shi jie ku ren duo.

Précisons, avant de voir le poème de Chisato (n. 13), que celui-ci n'avait pris comme sujet que le premier des deux vers/sujet chinois.

Toshi toki ni

masaru toshi nashi

to omoebaya

haru shimo tsuneni

sukunakaruramu
Ah, si l'on pouvait penser que dans une année, toutes les saisons se valent!

Le printemps ne serait pas toujours aussi court.

Le printemps, sous-entend le vers chinois, est la saison la plus agréable. C'est pourquoi il nous semble particulièrement court. Chisato a chanté cette même idée, en lui donnant «l'esprit» de son époque. On sent dans ce poème, dans la façon brillante de traiter le 
sujet (le conflit entre le poète et les lois de la nature qui affectent sa sensibilité), le style représentatif de la première anthologie impériale, le Kokinwakashû, qui sera compilée quelques années plus tard, vers 905, sur l'ordre de l'empereur Daigo.

Jien, dans son poème (n. 1919), a préféré un traitement plus allusif de la fugacité du printemps:

$\begin{array}{ll}\text { Kurete yuku } & \text { Le printemps qui s'achève } \\ \text { haru wa kasumi no } & \text { garde, de la brume } \\ \text { iro nagara } & \text { la couleur, } \\ \text { ayashiku nururu } & \text { et étrangement se mouille } \\ \text { hito no sode kana } & \text { la manche des gens. }\end{array}$

Le printemps a, dans le poème de Jien, la couleur de la brume. Or, la brume est un des attributs du printemps. Dire que le printemps a la couleur de la brume reviendrait à dire que l'hiver a la couleur de la neige, ou l'été celle du soleil. Une paraphrase du poème de Jien serait: alors que la saison arbore encore ses couleurs, ceux qui savent que le printemps s'achève versent des pleurs sans qu'il $\mathrm{y}$ ait de raison particulière. Jien a réussi dans son poème le tour de force de combiner les deux éléments des vers/sujet chinois; le passage trop rapide du printemps (sous entendu) et, par l'évocation des larmes, la souffrance des gens.

Examinons maintenant le poème de Teika (n. 419):

Itazura ni
haru hi sukunaki
hitotose no
taga itsuwari ni
kururu suga no ne
En vain,

les rares jours de printemps, dans une année (s'achèvent).

Qui donc en mentant (a dit qu'ils étaient longs) comme la racine de laîche qu'on tire?

Teika, à l'instar de Chisato, n'a retenu comme sujet que le premier vers du distique chinois. Il s'est donc limité dans son poème à l'évocation de la fugacité du printemps. Pour ce faire, il introduit dans son œuvre la laîche, plante aux longues racines emmêlées. La laîche apparaît dans la poésie japonaise classique pour évoquer quelque chose de long. Dans le Manyôshû, anthologie compilée au milieu du $\mathrm{VIII}^{\mathrm{e}}$ siècle, nous lisons (n. 1925): 
Ohohoshiku

kimi wo ahimite

suga no ne no

nagaki haru hi wo

kohiwataru kamo
En vous regardant

vaguement,

les longs jours de printemps, (longs comme) une racine de laîche, je les passerai amoureux.

Teika, dans la seconde moitié de son œuvre, attaque ceux qui, comme l'auteur du poème anonyme que nous venons de citer, parlent des «longs jours de printemps». C'est dans cette remise en cause d'une tradition anciennement établie que réside l'intérêt, l'originalité de son poème. Remarquons enfin que l'emploi du mot «laîche » pcrmct à Teika un jeu de mots sur le verbe kuru qui signifie à la fois «s'achever» (pour le jour, ou une saison), et «dévider», «tirer» (un fil ou quelque chose de long, ici la racine de laîche).

Voyons à présent les derniers poèmes de printemps de notre corpus. Ces poèmes ont été composés sur deux vers/sujets extraits du poème de Bai Juyi (livre 66, n. 3244) intitulé « Rendre visite au printemps, inscrit dans le jardin de M. Zhu », Xun chun ti zhu jia yuan lin.

IV - « Je vois une maison au loin avec des fleurs, j'entre donc (les admirer) / sans me soucier (de savoir si les gens qui l'habitent) sont nobles ou vils, aimables ou distants ", Yao jian ren jia hua bian ru / bu lun gui jian yu qin shu.

Examinons tout d'abord le poème de Chisato (n. 84):

Yoso nitemo

hana wo ahare to

miru kara ni

shiranu yado ni zo

mazu irinikeru
Même de l'extérieur, les fleurs, touchantes je les trouve, c'est pourquoi dans une maison inconnue, un instant je suis entré.

Chisato, qui n'avait pris pour sujet que le premier vers chinois, en a retenu les éléments que sont: "voir», "fleurs», «maison (inconnue)», «entrer» et «au loin» (rendu par «extérieur»), soit, la conjonction «donc» exceptée, tous les éléments du sujet. On peut aisément penser que Chisato avait à l'esprit, en composant son poème, les deux vers cités par Jien, car la «maison inconnue» de son poème 
peut être interprétée comme une façon adroite de rendre le second vers du sujet. Le thème du poème de Chisato n'en demeure pas moins celui du premier vers du sujet: les fleurs. Chisato justifie son goût pour celles-ci en les qualifiant de touchantes, ahare. Le ahare, ou émotion suscitée par l'impermanence, la fragilité des choses, est un concept central dans l'esthétique de l'époque de Heian. En ayant recours à ce terme, à cette perception des choses donc, Chisato «japonise » son œuvre.

Examinons à présent le travail de Jien sur ce même sujet (poème n. 1914):

Hana wo yado no aruji to tanomu haru nareba mini kuru tomo wo kirahu mono kawa
Je demande abri aux fleurs car c'est le printemps.

L'ami qui vient les voir, pourraient-elles le détester?

Jien a dans son poème opéré un changement d'angle. Le personnage était dans le vers chinois l'actant, et les fleurs, l'objet. Jien a choisi - et là réside l'intérêt de son poème - de personnifier les fleurs. Ce sont elles qui, par leur splendeur, donnent l'impression d'être le maître des lieux, et c'est à propos de leur sentiment qu'il s'interroge.

Voyons enfin le poème de Teika (n. 414):

Haruka naru
hana no aruji no
yado toeba
yukari mo shiranu
nobe no waka kusa

Haruka naru

yado toeba

nobe no waka kusa
Lorsqu'à la maison lointaine du propriétaire des fleurs je demande un abri, (je vois dans le jardin) ignorant le rapport (qui les lient aux maîtres des lieux), les jeunes herbes de la plaine.

Teika a gardé dans son poème les éléments du sujet que sont : la «maison», «les fleurs», et l'adjectif «lointain». Dans le vers/sujet, le poète s'introduisait dans un jardin sans se soucier de la qualité des propriétaires. Ce sont, dans le poème de Teika, des herbes sauvages (herbes de la plaine) qui croissent dans le jardin, ignorantes du lien (du rapport) qui les unit $\mathrm{au}(\mathrm{x})$ propriétaire(s). Teika a exprimé de cette 
manière détournée (et fort elliptique) l'indifférence à l'égard des habitants, que contenait le vers/sujet.

Les œuvres que nous allons examiner à présent sont des poèmes d'automne composés sur deux vers (un seul dans le cas de Chisato), extraits du poème de Bai Juyi (livre 14, n. 742) intitulé «Nuit de fin d'automne», Wan qiu ye:

$\mathrm{v}$ - «Au vol des oies du froid qui s'en retournent pressées, je comprends que l'automne s'achève / Le coq des voisins chante tard, je sais que les nuits sont longues », Sai hong fei ji jue qiu jin / Lin ji ming chi zhi ye yong.

Sur le premier vers donc, Chisato composa (n. 55):

Yuku kari no

tobu koto hayaku

mieshi yori

aki no kagiri to

omohishiriniki
Depuis que j'ai vu que les oies migratrices volaient rapidement, j'ai compris que c'était la fin de l'automne.

La similitude du poème de Chisato avec le vers chinois est, dans cet exemple, particulièrement frappante. Le poète n'a introduit dans son œuvre aucun élément nouveau significatif, aucune image. La seule différence entre les deux œuvres réside dans l'emploi par Chisato du verbe « voir».

Voyons maintenant le poème de Jien (n. 1942):

\author{
Ikani sen \\ yowa ni mataruru \\ tori no ne wo \\ isoganu aki to \\ omowamashikaba
}

\section{Comment faire?}
Ah, si seulement le chant des oiseaux, que dans la nuit je me trouve à attendre, pouvait me faire penser que l'automne n'est pas pressé.

Jien s'est efforcé dans son poème d'introduire le plus d'éléments possibles des vers/sujets; «la nuit», «l'automne», «pressé» (appliqué ici non plus aux oiseaux, mais à la saison). Dans les vers chinois apparaissaient les oies sauvages et le coq. Jien lui, parle dans son poème d'oiseau(x). S'agit-il d'oies ou de coq? Il laisse judicieusement 
le lecteur en décider. Le passage des oies et le chant tardif du coq sont dans les vers/sujet des signes de la fin de l'automne. Le poète les déchiffre et les accepte. Jien, dans son poème, se révolte contre l'inéluctabilité du signe, contre l'implacable passage du temps. Il souhaiterait - tout en sachant que cela est impossible - pouvoir changer la signification du signe, se leurrer un instant.

Teika, dans son poème (n. 442), choisit une tout autre approche du sujet :

\author{
Maki no ya ni \\ tonari no shimo wa \\ shirotahe no \\ yuhuzuke dori wo \\ itsuka kikubeki
}

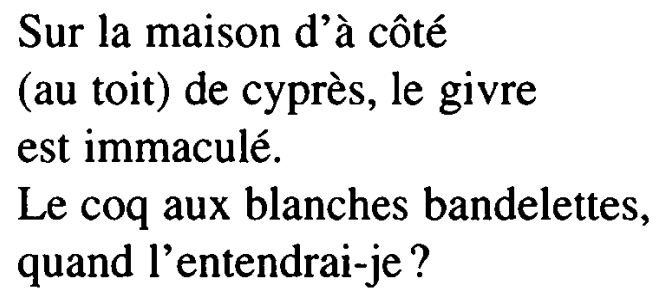

Teika a rejeté l'image des oies sauvages et signifié la fin de l'automne par l'évocation du givre qui, en poésie, marque la fin de la saison et le début de l'hiver. Il s'est dans son œuvre concentré sur le second vers du sujet, et, en retenant les éléments que sont la maison des voisins et le coq, a composé une symphonie de blanc. Le givre sur le toit est, selon ses propres termes, d'un blanc immaculé, tout comme le coq. Pour le nommer, Teika utilise le mot yuhuzuke dori qui, à l'époque ancienne, désignait un coq sur lequel on avait fixé (afin d'obtenir la faveur des dieux) des bandelettes blanches faites de fibres d'écorce. À l'époque de Teika ce terme ne désigne plus en poésie l'oiseau paré, mais tout simplement un coq. La blancheur des bandelettcs, implicite, est toutefois utilisée par l'auteur pour rappeler celle du givre. Teika a par ailleurs chanté les «longues nuits» du sujet de façon détournée, en s'interrogeant sur le moment où le coq allait chanter (et donc le jour se lever).

Les poèmes que nous allons examiner à présent furent composés sur deux vers de Bai Juyi extraits de son poème (livre 57, n. 2717) intitulé: "Cinquante rimes composées en se souvenant d'un voyage à l'est», Xiang dong you wu shi yun: 
VI - Ce monde d'illusions, un rêve (de nuit) de printemps / La vie éphémère, de l'écume sur l'eau.

Huan shi chun lai meng / Fu sheng shui shang ou.

Chisato composa un poème sur chacun des deux vers ${ }^{12}$. Tout d'abord, sur le premier (poème n. 112):

$\begin{array}{ll}\text { Maboroshi no } & \text { En mon cœur } \\ \text { mi toshi shirinuru } & \text { qui sait que je suis } \\ \text { kokoro ni wa } & \text { illusion, } \\ \text { hakanaki yume to } & \text { (ce monde), à un rêve éphémère } \\ \text { omohoyurukana } & \text { fait penser! }\end{array}$

Chisato a retenu du vers/sujet «l'illusion» et le «rêve». Un rêve (de nuit) de printemps est, dans les traditions chinoise et japonaise, un rêve bref, fragile, à cause de la fugacité des nuits de cette saison. Le vers chinois se lit comme une définition du monde, le poète s'efface derrière elle. Chisato, dans son œuvre, a introduit et même imposé sa présence par l'emploi des termes mi (corps, moi), et kokoro, (cœur, esprit). Le poème de Chisato est en quelque sorte l'acceptation, à titre personnel, de la vision du monde énoncée par le vers/sujet.

Voyons maintenant le poème (n. 113) qu'il composa sur le second vers :

$\begin{array}{ll}\text { Karisome ni } & \text { Notre esprit } \\ \text { shibashi ukaberu } & \text { qui, par hasard, } \\ \text { tamashihi no } & \text { flotte un instant, } \\ \text { mizu no awa tomo } & \text { à l'écume sur l'eau } \\ \text { tatoheraretsutsu. } & \text { est comparé. }\end{array}$

Notre esprit, qui doit sa brève existence au hasard, est, dit Chisato, comparé à l'écume. Chisato a retenu du sujet (le second vers) «l'eau», «l'écume » (image souvent employée pour exprimer l'éphémérité, la fragilité) et «flotter». Bai Juyi utilisait dans son poème le terme fusheng (en japonais fusei) «vie flottante» qui signifie une vie éphémère. Chisato a dans son œuvre remplacé ce terme par «esprit», tamashihi (et réutilisé le verbe flotter pour l'appliquer à l'écume). Ce changement fut vraisemblablement opéré par Chisato, car le mot «vie flottante» n'est pas employé en poésie. 
Examinons à présent le poème de Jien (n. 1999), qui avait pris comme sujet les deux vers de Bai Juyi:

Tonikakuni

ukiyo wo haru no

yume zo tomo

mizu no ahare $n i$

omohishiretoya
Que de toutes façons

ce monde flottant

est un rêve de printemps

grâce à la mélancolie de l'eau et à son

écume, comprenez-le!

Jien a combiné dans son poème le «monde» du premier vers/sujet et le «flottant» du second en «monde flottant», ukiyo, (qui signifie également «monde d'affliction"). Jien utilise-là une métaphore attestée au début $\mathrm{du} \mathrm{X}^{\mathrm{e}}$ siècle au Japon, et sans doute calquée sur l'expression chinoise fusheng, "vie flottante» (employée par Bai Juyi dans son vers/sujet) ${ }^{13}$. Jien a également retenu du sujet le «rêve de printemps» et «l'écume sur l'eau». L'écume était dans le vers chinois une métaphore pour la vie éphémère. Elle est chez Jien le révélateur de cette éphémérité. Notons enfin que Jien, jouant sur la similitude phonique de $a w a$, écume, et ahare, émotion, mélancolie, propose une double lecture de son quatrième vers (écume de l'eau/ mélancolie de l'eau).

Voyons maintenant le poème de Teika (n. 499):

Fuchi to naru

shigarami mo naki

hayasegaha

ukabu minawa zo

kiete kanashiki
Point de barrage de branchages

pour faire un creux profond dans la rivière au courant rapide, et l'écume flottant sur l'eau disparaît, que c'est triste !

Teika a rejeté le «monde d'illusion » et le «rêve d'une nuit d'été». Il s'est donc concentré pour sa composition sur le second vers/sujet. Il a retenu du sujet l'image de l'eau et de l'écume, et bâti tout son poème autour de la métaphore de la rivière, qui symbolise le cours du temps que rien ne peut retenir, et de l'écume qui, elle, exprime la vie éphémère.

Les dernières œuvres de notre corpus, des poèmes de plaintes, furent composées sur deux vers de Bai Juyi (un seul dans le cas de Chisato), extraits du poème (livre 54, n. 2469) intitulé : «Festin nocturne sur la muraille», Cheng shang ye yan. 
VII - «À ce que l'on dit, tout dans la vie n'est que rêve / Dans le rêve aussi, la joie l'emporte sur la tristesse », Cong dao zhu ren dou shi meng / Meng zhong huan xiao yi sheng chou.

Sur le second vers, Chisato composa (n. 116):

Yume nitemo

ureshiki koto no

mietsuru wa

tada ni urefuru

mi ni ha masareri
Même (si c'est) en rêve, avoir pu voir quelque chose d'heureux vaut mieux pour moi que de simplement souffrir.

Chisato a retenu tous les éléments du sujet chinois. La seule différence entre les deux œuvres réside dans l'emploi par Chisato des mots " pour moi », qui réduisent la portée universelle du vers/sujet en une considération personnelle.

Examinons le poème de Jien (n. 1986):

Utsutsu kamo

aru ka kokoro no

arigaho ni

yume ni yume miru

mi to ha shirazu ya
(Ce monde) est peut-être réalité, simule le cœur, mais l'être ne sait-il pas que tout est, dans un rêve, vision de rêve?

Jien n'a retenu du vers chinois que «le rêve». Ce mot lui permet de composer un poème profondément bouddhiste dans lequel s'opposent rêve, yume, et réalité, utsutsu, / cœur, kokoro, et corps, être, mi. (Précisons que kokoro, que nous avons traduit par «cœur», désigne également la volonté, l'intention, la pensée et l'esprit.)

Voyons maintenant le poème de Teika (n. 486) composé sur le même sujet:

\section{Ohokata no}

ukiyo ni nagaki

yume no uchi mo

kohishiki hito wo

mite ha tanomaji
Dans le long rêve

de ce monde de tristesse

ordinaire,

même si je vois l'être que j'aime, je ne m'y fie point. 
Teika n'a retenu comme élément des vers/sujets que le rêve, qui est ici encore une métaphore pour la vie. Les vers de Bai Juyi dénotaient un profond optimisme. Celui-ci a complètement disparu de l'œuvre de Teika, pour qui l'amour même pourrait n'être qu'illusion.

Après avoir examiné brièvement chacun des poèmes de notre corpus, il convient à présent d'essayer d'extraire de ces exemples les règles qui sous-tendaient ce mode de composition. Pour ce faire, tentons avant tout de synthétiser l'attitude du poète face à son matériau : le vers/sujet chinois. Remarquons tout d'abord que les vers/sujets choisis traitent tous des thèmes classiques de la poésie chinoise. Les images mises en œuvre dans ces vers sont également traditionnelles. Les sujets peuvent être décomposés en deux unités : les éléments (les caractères) qui les constituent, et le sens global du (des) vers. Le poète japonais dosera la quantité de chacune de ces unités qu'il désire introduire dans son œuvre. Dans le cas de Chisato, nous avons pu remarquer que l'emprunt aux éléments du sujet était maximal. Quels termes Chisato a-t-il donc rejetés? Dans ses compositions de notre corpus, nous n'avons pu relever qu'un seul rejet : la «beauté du paysage» (sujet II) que Chisato a remplacée par « le vent à la couleur profonde ». Ce rejet résulte-t-il du désir de Chisato de se démarquer de son modèle chinois? Le caractère unique de l'exemple nous invite à penser que non. Le choix de Chisato fut sans doute davantage guidé par les nécessités de la métrique japonaise et/ou de l'harmonie des sons. Nous pouvons donc supposer que pour Chisato, composer sur un vers/sujet chinois signifie réutiliser, autant que possible, tous les éléments qui le constituent.

Si l'on examine à présent les poèmes de Jien et Teika, nous remarquons une attitude différente de ces deux poètes vis-à-vis du sujet. Nous avons pu remarquer tout d'abord que Jien avait parfois choisi comme sujet pour un waka plusieurs vers chinois. Cela est possible au début du XIII ${ }^{\mathrm{e}}$ siècle, car on n'exige plus du poète qu'il introduise tous les termes du vers/sujet. Le poète doit opérer un choix et ne retenir dans son poème que les termes qu'il juge nécessaires. Pour Jien et Teika - comme pour tous les poètes de leur temps sans doute composer sur un vers chinois revenait à composer sur un sujet constitué de plusieurs éléments (en japonais musubi dai ${ }^{14}$ ). Au début 
$\mathrm{du} \mathrm{XII}^{\mathrm{e}}$ siècle, le poète et critique Minamoto no Toshiyori écrivait dans son traité intitulé Toshiyori zuinô :

D'une manière générale, pour composer un poème, il faut bien comprendre le sujet. Que le sujet soit composé de trois, quatre ou cinq éléments, peu importe. Il faut bien savoir quels sont les éléments à introduire (dans son poème), ceux qu'il ne faut à aucun prix reprendre, ceux dont il faut suggérer le sens, ceux qu'il faut absolument faire apparaître (dans le poème). Les éléments dont le sens doit être simplement suggéré, il est mauvais de les faire apparaître (dans le poème). Il est également mauvais de (simplement) suggérer les éléments qui doivent apparaître (dans le poème), car le sujet perd alors de son unité. Ceci ne se transmet pas (de maître à élève). Il faut le comprendre de soi-même ${ }^{15}$.

Toshiyori dresse là un inventaire des différentes possibilités de traiter un élément du sujet: ne pas en tenir compte, le reprendre tel quel, ou encore de façon indirecte, détournée. Il précise que la façon de traiter chacun des éléments du sujet ne s'enseigne pas, que le poète (expérimenté) la saisira de lui-même. Cela signifie, en d'autres termes, qu'il n'existe pas de règle fixe et que c'est le sens poétique, le «bon goût » du poète qui décide. Cette absence de règle fixe explique les différences sensibles entre les choix de nos trois poètes. Un relevé pour chaque sujet des éléments retenus en commun chez nos trois auteurs fait apparaître le résultat suivant:

Sujet n. I : convier, rossignol, sous, fleurs (de prunier).

Sujet n. II : fleurs (de prunier).

Sujet n. III : jours de printemps.

Sujet n. IV : maison, fleurs.

Sujet $\mathrm{n} . \mathrm{v}$ : automne (exprimé de façon indirecte chez Teika).

Sujet n. VI : écume, eau.

Sujet n. VII : rêve.

Si l'on relève les éléments du sujet qui ne se retrouvent, en commun, que dans les poèmes de Chisato et Jien, on obtient le résultat suivant : 
Sujet n. I : néant.

Sujet n. II : oublier de rentrer (exprimé de façon détournée chez Jien).

Sujet n. III : néant.

Sujet n. IV : néant.

Sujet n. v : oies («oiseaux » chez Jien).

Sujet n. VI : rêve.

Sujet $\mathrm{n}$. VII : néant.

Relevons enfin les éléments du sujet qui ne se retrouvent en commun que dans les poèmes de Jien et Teika:

Sujet n. I : néant.

Sujet n. II : sous.

Sujet n. III : néant.

Sujet n. IV : néant.

Sujet n. v : coq («oiseau » chez Jien).

Sujet n. vi : néant.

Sujet $\mathrm{n}$. vII : néant.

Ces résultats nous enseignent que le(s) seul(s) élément(s) du vers/sujet que le poète doit absolument introduire dans sa composition sont:

1 - Dans le cas de poèmes de saison, les mots qui permettent de reconnaître immédiatement quelle est la saison chantée dans le poème. Ces mots, appelés en japonais kigo «mots de saison», sont par exemple, pour le printemps les fleurs de prunier, le rossignol; pour l'été, l'oranger sauvage, les pluies du cinquième mois (samidare); pour l'automne, les oies migratrices, le givre, et enfin pour l'hiver, la neige et la glace. (Nous avons toutefois remarqué, au sujet $n$. $v$, que pour les nécessités de la composition - une symphonie de blanc -, Teika a remplacé les kigo du sujet par d'autres termes évoquant la même saison.)

2 - Dans le cas de poèmes composés sur des sujets autres que les saisons, les poètes ont retenu un ou plusieurs «mots-clés». (Ainsi, pour le sujet $n$. VII qui servit à la composition de poèmes de plaintes, nos trois poètes retinrent le mot «rêve ».) $\mathrm{Ce}(\mathrm{s}) \operatorname{mot}(\mathrm{s})$-clés furent 
intégrés dans un poème dont le sens exprimé était celui de la section poétique à laquelle il appartient (plaintes, impermanence etc).

Après avoir examiné les éléments du sujet que les poètes ont retenus dans leurs œuvres, il convient de nous pencher un instant sur ceux qu'il ont rejetés. Nous avons remarqué tout d'abord que, dans tout notre corpus, un seul élément avait été rejeté par nos trois poètes. Il s'agit de la "beauté du paysage » du sujet n. II. Nous pouvons expliquer ce rejet par deux raisons; d'une part la difficulté déjà soulignée de traduire de façon harmonieuse le terme, et d'autre part, par le fait que le sujet évoque les pruniers en fleur, ce qui, pour les Japonais, sous-entend qu'il s'agit d'un beau paysage. Introduire le «beau paysage » eût sans doute été redondant.

Nous remarquons par ailleurs que Chisato a rejeté très peu d'éléments du sujet (« sous » du sujet $\mathbf{n}$. II, et « monde » du sujet $\mathrm{n}$. VI). Rappelons ici que dans quatre cas sur sept (sujets III, IV, v, vII) Chisato n'avait pris comme sujet que la moitié des vers retenus par Jien. Le choix à opérer parmi les éléments était donc moindre. Jien et Teika, en revanche, ont à plusieurs reprises rejeté la moitié du sujet, quand les éléments qu'elle contenait ne pouvaient être intégrés de façon naturelle dans leur œuvre. (Jien : sujets IV, VII, Teika : sujets III, V, VI). Jien - qui avait choisi les sujets - s'est plus souvent que Teika efforcé d'introduire les éléments des deux vers.

Si l'on fait le décompte, sujet par sujet, des éléments rejetés par Jien, nous obtenons les résultats suivants :

Sujet n. I : deux éléments sur 6.

Sujet n. II : deux éléments/5.

Sujet $n$. III : quatre éléments/6.

Sujet n. IV : deux éléments $/ 5+$ la moitié du sujet $=7 / 10$.

Sujet $n . v$ : sept éléments/12.

Sujet $n$. vi : deux éléments/7.

Sujet $n$. VII : un éléments $/ 4+$ la moitié du sujet $=6 / 9$.

Le même décompte chez Teika:

Sujet $\mathrm{n} . \mathrm{I}$ : néant $/ 6$.

Sujet n. II : trois éléments/5. 
Sujet $n$. III : un élément $3+$ la moitié du sujet $=4 / 6$.

Sujet n. IV : un élément/10 (tout le second vers du sujet ayant été exprimé de façon détournée).

Sujet $n$. $v$ : trois éléments/12 (dont la moitié du sujet).

Sujet $n$. VI : quatre éléments/7 (dont la moitié du sujet).

Sujet n. VII : quatre/9.

Ces résultats laissent apparaître un rejet d'éléments du sujet légèrement plus important chez Jien que chez Teika (et considérablement plus important chez ces deux poètes que chez Chisato).

Un examen des éléments ayant été rajoutés par nos poètes dans leurs compositions révèle que Chisato a très peu opéré d'ajouts (nous ne relevons que trois éléments qui ne faisaient pas partie du sujet, introduits par Chisato dans ses œuvres: sujet $n$. II « le vent à la couleur profonde», sujet n. IV, l'adjectif «touchant», et enfin pour le sujet n. VI, le mot «cœur»). L'introduction de nouveaux éléments est en revanche abondamment pratiquée par Jien et Teika. Chacun de leurs poèmes contient au moins un élément nouveau, et parfois même trois (par exemple les poèmes composés sur le sujet $n$. VII). Le rajout de nouveaux éléments résulte-t-il d'un simple désir d'exprimer différemment le sens du sujet, ou au contraire d'une volonté de se démarquer de celui-ci, d'exprimer autre chose? C'est ce à quoi devrait nous permettre de répondre l'examen de la signification des poèmes composés par nos trois auteurs.

L'examen des poèmes de notre corpus au regard de leur sujet chinois nous permet de dégager deux grandes tendances pouvant être subdivisées ainsi :

1-a- Le sens du vers/sujet et sa conception ont été conservés.

1-b- Le sens du vers/sujet a été conservé, mais la conception modifiée.

2-a- Le sens du vers/sujet a été modifié, mais la conception conservée.

2-b- Le sens du vers/sujet et la conception ont été modifiés.

Si l'on classe tous les poèmes de notre corpus selon cette grille, nous obtenons le résultat suivant. $(\mathrm{C}=$ Chisato, $\mathrm{J}=\mathrm{Jien}, \mathrm{T}=$ Teika $)$ 


\begin{tabular}{|c|c|}
\hline 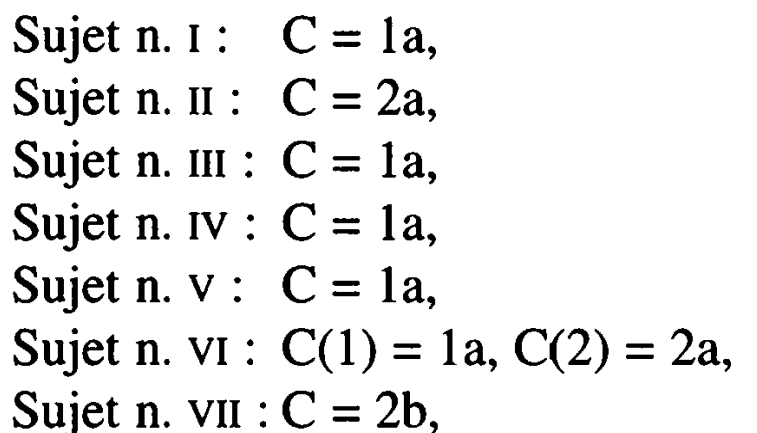 & $\begin{array}{l}\mathrm{J}=2 \mathrm{~b}, \\
\mathrm{~J}=2 \mathrm{~b}, \\
\mathrm{~J}=2 \mathrm{~b}, \\
\mathrm{~J}=2 \mathrm{~b}, \\
\mathrm{~J}=2 \mathrm{~b}, \\
\mathrm{~J}=2 \mathrm{a}, \\
\mathrm{J}=2 \mathrm{~b},\end{array}$ \\
\hline
\end{tabular}

Ces résultats révèlent que Chisato $a$, dans la plupart des cas, conservé à la fois le sens et la conception (l'ordre et la formulation des images) du vers/sujet. Sur les trois exemples de poèmes de Chisato qui diffèrent de leur sujet chinois, deux doivent cette différence au remplacement d'un élément du sujet par un autre («beauté du paysage » par «vent à la couleur profonde» au sujet $n$. II, et «la vie» par «l'esprit» au sujet $\mathbf{n}$. VI). Seul le poème composé sur le sujet $\mathbf{n}$. VII, révèle, du fait que l'auteur prend à son compte la vérité universelle énoncée dans le sujet, une transformation réelle (bien que minime) du sens du vers/sujet.

Les poèmes de Jien et Teika, en revanche, révèlent presque tous ${ }^{16}$ un double travail opéré sur le sens du vers/sujet et sur la conception du nouveau poème. Cette différence dénote une évolution des règles de composition. Le recueil de Chisato fut composé, rappelons-le, en 894 ou 896. À cette époque, la poésie japonaise, pourtant pratiquée par toute la cour, ne jouissait pas officiellement au Japon du même prestige que la poésie chinoise. Il faudra attendre la compilation et la présentation à l'empereur en 905 de la première anthologie de poésie japonaise compilée sur ordre impérial, le Kokin wakashû, Recueil de poèmes japonais anciens et modernes, pour que la poésie japonaise ne soit plus considérée comme étant inférieure à la chinoise. Le Kokin wakashû est sans doute l'aboutissement d'un long travail de réflexion sur la poésie japonaise, mené par différents poètes, dont $\mathrm{Ki}$ no Tsurayuki ${ }^{17}$. La question de la supériorité supposée de la poésie chinoise devait intéresser bien des poètes de l'époque. Nous sommes de ce fait en droit de lire le travail de Chisato comme une des pierres apportées à l'édifice de la poésie japonaise. En produisant des poèmes japonais composés sur des vers/sujets chinois, Chisato ne visait sans doute nullement l'originalité. Ses compositions prouvaient que l'on 
pouvait exprimer les même images, les mêmes émotions que celles contenues dans les vers chinois, par le truchement de la poésie japonaise. Son travail, le premier de son genre rappelons-le, prouvait également qu'un poème japonais n'est en rien inférieur à un poème chinois. C'est un travail de pionnier, de militant, qu'accomplit Chisato.

Au début du XIII ${ }^{\mathrm{e}}$ siècle, les données sont tout à fait différentes. Le waka jouit depuis plusieurs siècles déjà d'un statut prestigieux, il n'est plus nécessaire de conforter sa position. Si Jien a choisi des vers de Bai Juyi comme sujets de composition, c'est pour exprimer son admiration à l'égard de ce grand poète qu'il considère comme «l'incarnation de Mañjusrî ${ }^{18}{ }$, bodhisattva parangon de la Sapience. Pour Jien, comme pour Teika, composer sur des vers de Bai Juyi, c'est produire des variations sur un thème donné, jouer avec l'intertextualité.

Jien et Teika, contrairement à Chisato, se sont efforcés, en partant du vers/sujet, de produire un sens nouveau. Ils ne font là qu'obéir aux impératifs de la poétique de leurs temps, qui imposait la recherche de la nouveauté dans la conception du poème, afin d'éviter la sclérose du waka dont le vocabulaire et les images sont strictement codifiés.

Ces quelques considérations nous invitent à nous interroger maintenant sur l'apport du kudai waka. Les poèmes composés sur des vers/sujets chinois ont-ils quelque chose de particulier? En règle générale, non. Les poèmes japonais que nous avons analysés ne présentent aucune «couleur chinoise». Nous avons remarqué plus haut que les vers/sujets cités présentaient des thèmes traditionnels de la poésie chinoise. Il convient de préciser maintenant que ces thèmes étaient tous, à l'époque de Chisato, déjà couramment utilisés dans la poésie japonaise, dont ils constituent également des thèmes classiques. Il serait donc vraisemblablement impossible de déceler le mode de composition des waka de notre corpus, si les vers chinois qui servirent de sujet n'étaient pas consignés en regard. L'utilisation de vers chinois ne relève donc aucunement d'un désir d'exotisme. Il s'agit plutôt, audelà de l'hommage à un poète admiré ou du désir de se mesurer à une œuvre (re)connue, d'un moyen d'obtenir de nouvelles combinaisons d'images, de nouveaux effets, de renouveler les gammes de couleurs sur lesquelles composer. Le kudai waka était aussi selon toute vraisemblance un exercice permettant au poète de tester sa virtuosité. 
Chisato, après avoir reposé son pinceau, offrit son recueil de poèmes à l'empereur Uda. Le rang du destinataire nous permet de supposer que Chisato produisait là le meilleur de lui-même. Jien, une fois les séquences achevées, les présenta en offrande au sanctuaire de Kitano, dédié à Sugawara no Michizane, poète qui composait comme nous l'avons mentionné au début de cet article en chinois et japonais et qui est considéré comme le dieu protecteur des lettres. Ce faisant, il exprimait son admiration pour le poète et, à travers lui, pour les deux poésies, chinoise et japonaise. Il inclut ensuite son travail dans l'anthologie de ses poèmes personnels, le Shûgyoku shû. Il s'agissait donc pour Jien d'œuvres poétiques à part entière. 'leika, lui, classa sa séquence dans l'annexe de son anthologie personnelle, appelée Shûigusô ingai. Par ce geste il montrait qu'il ne souhaitait pas voir ses poèmes composés sur des vers/sujets chinois figurer au sein même de son anthologie personnelle, et, en d'autres termes, qu'il s'agissait là pour lui avant tout d'une «expérience poétique».

\section{NoTES}

* L'auteur de cet article tient à exprimer sa profonde reconnaissance, à la fois à Mlle Jacqueline Pigeot qui a relu son texte et lui a indiqué de nombreuses corrections à apporter, et à M. François Martin, qui a consacré bien du temps à guider ses pas dans la lecture et la traduction des vers de Bai Juyi.

1. Dates de naissance et de mort inconnues. Issu d'une famille de spécialistes d'études confucianistes, Chisato essaya sans succès de tirer parti de cet atout pour faire carrière. (Le poste le plus élevé qu'il obtint fut celui de «fonctionnairc principal du département des affaires militaires ", hyôbu no daijô, en 903.) L'œuvre la plus importante qui nous ait été conservée de Ôe no Chisato est son recueil personnel de poèmes japonais, Chisato shû. (126 poèmes). Il ne subsiste malheureusement pratiquement aucun exemple de composition en chinois de ce poète qui écrivait dans les deux langues, chinoise et japonaise.

2. Le waka, « poème japonais » (appelé également tanka «poème court », ou encore $u t a$, «chant») est composé de trente-et-une syllabes réparties en cinq vers ou $k u$, de 5/7/5/7/7 syllabes respectivement.

3. HoNMA, 1992, 181.

4. Shinpen kokka taikan, vol. 3, p. 868.

5. Il suffit pour s'en convaincre de lire la monumentale étude de KANEKo Hikojirô, Heian jidai bungaku to Hakushi monjû. 
6. Jien, fils du grand chancelier Fujiwara no Tadamichi, est issu de la haute noblesse. En 1167, il se retire du monde et, en 1192, devient recteur monacal surnuméraire, gon no sôjô, et supérieur de la secte Tendai, Tendai zasu. Parallèlement à sa carrière de moine, il s'illustre comme poète de waka, participant aux différentes activités poétiques de la cour (nous ont été conservés quelques six mille de ses waka). Jien est également l'auteur du premier essai d'historiographie japonaise, le Gukanshô.

7. Des séquences de cent poèmes furent fréquemment composées au Japon à partir de la fin du $x^{e}$ siècle. (Le plus ancien exemple connu est celui composé par Sone no Yoshitada, vers 960.)

8. Fils du grand poète Fujiwara no Shunzei (alias Toshinari), Teika (alias Sadaie) mena lui aussi une brillante carrière de poète. En plus d'une œuvre poétique abondante et d'exceptionnelle qualité (son recueil personnel contient plus de quatre mille waka), Teika écrivit également des traités de poétique qui le consacrèrent d'emblée comme le plus grand théoricien de la poésie de son temps. Teika est par ailleurs le seul poète de l'histoire de la poésie japonaise à avoir eu l'insigne honneur de participer à la compilation de deux anthologies impériales de waka.

9. Le moine poète Jakushin (1191-1251) composa également sur les mêmes sujets. Toutefois, quarante poèmes seulement nous ont été conservés (dans son recueil personnel), et les conditions de composition restent obscures.

10. La numérotation des poèmes de Bai Juyi suit celle de l'index Hakushi Monjû sakuin établi par HIRAOKa Takeo et IMAI Kiyoshi.

11. La numérotation et la transcription des waka suit celle des anthologies personnelles de nos trois auteurs contenues dans le vol. 3 du Shinpen Kokka taikan.

12. Signalons que Chisato a rangé ses deux poèmes dans la section «plaintes", jukkai, de son anthologie. Les compositions de Jien et Teika figurent, elles, dans la section «impermanence », mujô, de leurs séquences.

13. Sur l'origine du terme ukiyo, on lira Bernard FRANK, annuaire E.P.H.E, IV section, 1966-67.

14. Le vocabulaire technique poétique classe les sujets de poèmes en deux grandes catégories : "sujets simples", qui ne comprennent qu'un seul élément, en japonais hitomoji dai (par exemple «printemps» «fleurs de cerisiers» etc.) et «sujets liés», musubi dai, quand le sujet comprend deux éléments, ou plus (par exemple «pluie de printemps», «saule pleureur dans la neige » etc.).

15. Toshiyori zuinô, in Karon-shû, p. 83.

16. Seul le poème de Teika composé sur le sujet $n$. III reprend à l'identique le sens du vers, mais en modifie la conception.

17. Ki no Tsurayuki (?-945). Lié à Ôe no Chisato avec qui il participa à diverses manifestations poétiques, Ki no Tsurayuki est l'une des personnalités littéraires les plus importantes de son temps. Poète admiré, il fut l'un des compilateurs de la première anthologie de waka compilée sur ordre impérial, le Kokin waka shûu dont il écrivit la préface en japonais (il existe également une préface écrite en 
chinois par $\mathrm{Ki}$ no Yoshimochi). Ce texte est considéré comme le premier véritable essai de poétique japonaise.

18. Nous trouvons cette considération dans la post-face du Monjû hyakushu, p. 685 , du vol. 3 du Shinpen kokka taikan.

\section{BIBLIOGRAPHIE}

\section{Édition des sources :}

(Ouvrage collectif), Shinpen kokka taikan, vol. 3, Shikashû hen, Kadokawa shoten, 1985,2 vol.

Hashimoto Fumio (annoté par), Shunrai zuinô, in Karonshû, col. Nihon koten bungaku zenshû vol. 50, ed. Shôgakkan, 1975.

HiraOKa Takeo et Imal Kiyoshi, Hakushi monjû sakuin, Dohosha, 1989, 2 vol.

KuBota Jun, Yaku chû-Fujiwara no Teika zenkashû, Kawade shobô shinsha, 1986, 2 vol.

OKamura Shigeru, Hakushi monjû, col. Shinshaku kanbun taikei (vol. 99,100,103), ed. Meiji shoin, 3 vol. parus (vol. III, 1988, vol. IV, 1990, vol. VI, 1993).

\section{Références bibliographiques :}

Honma Yôichi, « Kudai waka no sekai », Ronshû, Dai no waka kûkan, Kazama shoin, 1992, p. 179-202.

Kaneko Hikojirô, Heian jidai bungaku to Hakushi monjû, Baifû kan, 1943, réed. $1955,884+44$ p.

NaGATANI Kanji, «Monjû hyaku shu no kenkyû », Baika joshi daigaku bungakubu kiyô, $\mathrm{n}^{\circ} 11$, déc. 1974 , p. 1-16, (première partie), $\mathrm{n}^{\circ} 12$, déc. 1975, p. 1-18 (seconde partie).

SATô Tsuneo, «Kenpô roku nen "Monjû hyaku shu" no seiritsu », Chûsei bungaku, juillet 1975, p. 1-13.

«Teika. Jien no Hakushi monjû juyô », Chûsei bungaku, n 18, 1973, p. 1-7. «Shi ku daiei ni okeru futatsu no taido, Monjû hyaku shu no Jien to Teika », Wakan hikaku bungaku sôsho, n. 5, Kyûko shoin, 1986, p. 25-46.

Monjû hyakushu hokô, Kagawa daigaku kyôiku gakubu, Kenkyû hôkoku dai ichi bu, vol. 40, 1976, p. 37-80. 